\title{
Trained by feeding
}

Circadian rhythms are synchronized in mammals by time of feeding and light exposure. The mechanisms of circadian entrainment by external light stimuli are well established; however, the effects of feeding cues are unclear. Research published in Cell now shows that the feedingassociated hormones, insulin and insulin-like growth factor 1 (IGF1), act as global synchronizing cues for mammalian circadian rhythms.

"As biological circadian rhythms are a cellular property, non-proliferating fibroblast cells are the ideal system for investigating fundamental questions about clock mechanisms," explains corresponding author John S. O'Neill. The authors observed that insulin treatment induced expression of the PERIOD clock protein PER2 in fibroblasts. "We saw the same effect in primary cells (cardiomyocytes and neurons), gut organoids and tissues ex vivo (lung and kidney)," says O'Neill. Importantly, insulin treatment in reporter mice elicited rapid increases in PER2 expression compared with control mice, implicating insulin as a circadian regulator in vivo.

Feeding cues were previously proposed to act on a hypothetical 'food-entrainable oscillator' (FEO), a neuronal locus that would translate environmental stimuli into behavioural changes. So far, research has failed to identify the FEO. By contrast, light stimuli are known to act through the hypothalamic suprachiasmatic nucleus (SCN). In the present study, the researchers found that the SCN did not respond to insulin treatment.

Interestingly, IGF1 was also found to induce PER2 expression in vitro. Moreover, PER2 expression could be induced in fibroblasts by insulin or IGF1 in the absence of glucose, suggesting that circadian entrainment is a new glucose-independent function of insulin signalling. In addition, pharmacological inhibition of insulin receptor and IGF1 receptor in reporter mice was associated with attenuated entrainment of circadian rhythms in response to changes in feeding time.

"The final interesting finding is that the mechanism of PER2 induction involves increased translation of pre-existing mRNA, rather than transcriptional induction, with three-way

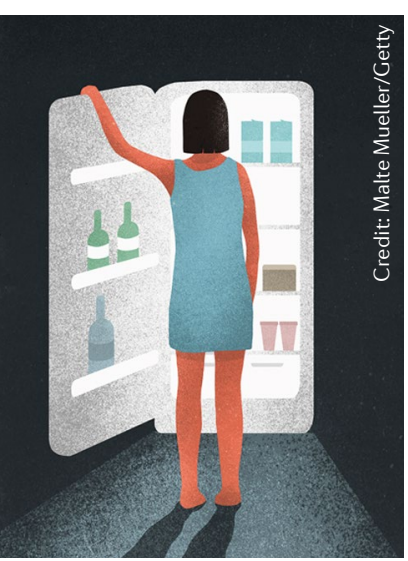
coincidence detection being required to affect the molecular clockwork (that is, mTORC activation, PTEN inhibition and per-cognate microRNA degradation)," explains O’Neill.

"We imagine this is important for allowing cells to discriminate timing signals from the large number of other signalling functions that are mediated by TOR complexes."

These findings suggest that the FEO does not exist and that, instead, insulin and IGF1 are feeding cues that act systemically to synchronize circadian rhythms.

Shimona Starling

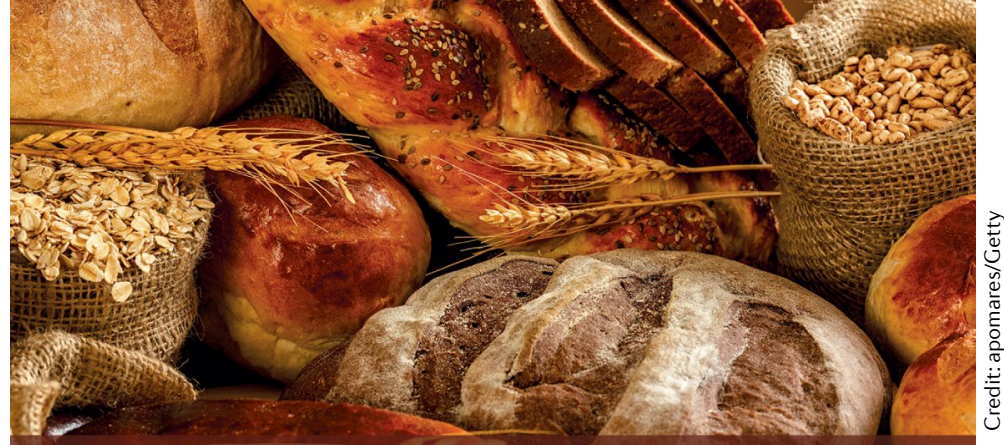

$\Rightarrow$ METABOLISM

\section{Metabolic safety of common preservative under scrutiny}

Small amounts of propionate, which is a common preservative found in food (particularly baked goods), can increase levels of blood glucose in humans, according to new research published in Science Translational Medicine. Amir Tirosh and colleagues found that just $1 \mathrm{~g}$ of calcium propionate (also known as E282) had a marked effect on the systemic level of key hormones, such as fatty acid-binding protein 4 (FABP4) and glucagon, in humans.

"This project stemmed from ongoing research on the metabolic actions of FABP4," explains Tirosh. "We came across a paper from 1912 where the authors showed that administration of propionate to dogs resulted in increased glucose production." Given the growing interest in this short-chain fatty acid, which is widely used as an ingredient in processed food, Tirosh and co-workers decided to repeat the 1912 study. "We wanted to investigate whether wild-type mice, FABP4deficient mice and mice lacking hepatic glucagon receptor respond similarly to propionate," adds Tirosh.

The team were able to replicate the findings from 1912 in wild-type mice, showing that treatment with oral propionate caused marked hyperglycaemia; however, by using FABP4-deficient mice and mice that lacked the hepatic glucagon receptor, they were able to study the differential contribution of FABP 4 and glucagon to this hyperglycaemic response. "We found that propionate stimulated glycogenolysis and hyperglycaemia in mice by increasing plasma concentrations of noradrenaline, glucagon and FABP4," adds Tirosh. "Furthermore, the hyperglycaemic response induced by propionate was dependent on FABP4."
Next, Tirosh and colleagues studied the translational potential of their findings to human physiology in a small randomized, placebo-controlled, double-blinded study. They gave 14 lean healthy volunteers a meal with or without the addition of $1 \mathrm{~g}$ of calcium propionate (equivalent to $0.3 \% \mathrm{w} / \mathrm{w}$, a common concentration used for the preservation of foods). The group collected blood samples before the participants ingested the meal and serial samples every 30 minutes for 4 hours after the meal had been eaten.

The authors found, just as they did in mice, that ingestion of propionate at levels similar to what is added to food for preservation increased plasma concentrations of noradrenaline, glucagon and FABP4, which in turn resulted in an increase in levels of blood glucose. "We were very surprised to find that even when a small amount of propionate was given to humans, it had a marked effect on the systemic concentration of these key hormones," explains Tirosh. "Noradrenaline, glucagon and FABP4 normally act during fasting to protect against a dangerous drop in blood glucose; in this case, however, they are engaging inappropriately and increasing blood glucose."

Tirosh and colleagues are continuing their research into the molecular components of food. "Such studies could lead to the identification of other ingredients that could be hazardous, but also some that might be beneficial, to metabolic health," concludes Tirosh.

Alan Morris

ORIGINAL ARTICLE Tirosh, A. et al. The shortchain fatty acid propionate increases glucagon and FABP4 production, impairing insulin action in mice and humans. Sci. Transl Med. 11, eaav0120 (2019) 